\title{
Effect of different light regimes on esterase isozyme profiles of three species of Drosophila
}

\section{Shereen Kouser, V. Shakunthala \& S. N. Hegde}

To cite this article: Shereen Kouser, V. Shakunthala \& S. N. Hegde (2013) Effect of different light regimes on esterase isozyme profiles of three species of Drosophila, Frontiers in Life Science, 7:3-4, 148-154, DOI: 10.1080/21553769.2013.867417

To link to this article: https://doi.org/10.1080/21553769.2013.867417

$$
\text { (c) } 2014 \text { Taylor \& Francis }
$$

\footnotetext{
曲 Published online: 23 Jan 2014.
}

Submit your article to this journal $\pi$

\section{Lll Article views: 179}

Q View related articles $₫$ 


\title{
Effect of different light regimes on esterase isozyme profiles of three species of Drosophila
}

\author{
Shereen Kouser*, V. Shakunthala and S. N. Hegde \\ Department of Studies in Zoology, University of Mysore, Manasagangotri, Mysore-06, Karnataka, India
}

(Received 10 July 2013; accepted 16 November 2013)

\begin{abstract}
Circadian rhythm has been identified in every organism studied, from unicellular marine algae to man, and in virtually all physiological and biochemical functions. The endogenous circadian system functions to organize behaviour and physiology to adapt to and anticipate environment changes in light and temperature. The present study is an attempt to understand enzyme profiles (alpha and beta esterases) of Drosophila melanogaster (Oregon-K strain), Drosophila gangotrii and Drosophila jambulina under light/dark (LD), continuous light (LL) and continuous dark (DD) conditions over 30 generations. A polyacrylamide gel electrophoresis $(7.5 \%$ - native gel) was used to study the esterase isozyme banding patterns in three species of Drosophila. It has been noticed that there were three alpha esterase loci in Drosophila species which were designated as $\alpha$-est $1, \alpha$-est 2 and $\alpha$-est 3. Similarly there were three beta esterase loci which were designated as $\beta$-est $1, \beta$-est 2 and $\beta$-est 3. Flies maintained in different light regimes showed differences in their allelic patterns with respect to alpha and beta esterases. It was observed that there was expression of some bands at a given light regime and the absence of the same in another regime. This shows that the different light regimes affect the expression of esterase isozymes.
\end{abstract}

Keywords: endogenous; physiology; esterase

\section{Introduction}

Circadian rhythms are daily cycles in the behaviour and physiology of an organism. The cycles are endogenously generated self-sustaining rhythms but can be influenced by environmental stimuli such as light and feeding (Schibler 2005). Circadian clocks in flies help to coordinate rhythmic feeding behaviour and regulate proper energy consumption and metabolism. Food consumption in Drosophila consistently occurs at specific times of the day (primarily during the morning), and this rhythmic behaviour persists under constant darkness (Xu et al. 2008). It has been shown that many organisms have evolved biological clocks to time events by metabolic processes rather than simply responding to the daily light/dark transitions. By using a circadian clock animals adjust their timing to the environment. Circadian clocks in different organisms use different sets of genes that are similar in terms of their function in the molecular feedback loops (Edery et al. 1994; Blau \& Young 1999). In addition, these clock genes also influence several vital metabolic cycles and are therefore believed to play an essential role in adaptive mechanisms (Dvornyk et al. 2003). The periodic analysis of certain (metabolic) enzymes which may directly influence the entrainment of an individual with respect to different light/dark periods can be studied to understand the changes with respect to their adaptation. If flies are subjected to different light regimes for several generations it may alter their circadian rhythmicity. Maintaining flies in different light regimes is nothing but subjecting the flies to stressful condition. There are reports that Drosophila flies showed difference in their fitness when subjected to different light regimes (Sheeba et al. 2000; Sharma 2003). It has been shown that in $D$. melanogaster the light/dark (LD) regime affects pre-adult development time (Sheeba et al. 1999), lifetime fecundity (Sheeba et al. 2000) and adult life span (Pittendrigh \& Minis 1972; Klarsfeld \& Rouyer 1998; Hendricks et al. 2003; Kumar et al. 2005). Studies have shown that Drosophila species have maximum fertility with LD exposure and minimum with continuous dark (DD) exposure (Harini 2010). It has also been shown that in D. melanogaster, D. gangotrii and D. jambulina maximum time took place for complete emergence in LD, whereas in continuous light (LL) clock is fasten up and in DD clock is slow hence flies showed delayed emergence when compared to LL (Shereen $\&$ Shakunthala 2012). But there are not many reports on the effect of different light regimes on esterase isozyme profiles of Drosophila with respect to their circadian clock.

Esterases are complex enzymes acting on a variety of substances and are capable of hydrolysing ester bonds. In insects, esterases are involved in important physiological processes, including the catabolism of juvenile hormone (Zera \& Holtmeir 1992; Shanmugavelu et al. 2000), juvenile hormone regulation (Hidayat \& Goodman 1994), insecticide resistance (Morton 1993), ontogenetic development

\footnotetext{
*Corresponding author. Email: shereenkouser@gmail.com
} 
(Bitondi \& Mestriner 1983), digestion (Argentine \& James 1995), functioning of the nervous system (Villatte \& Bachmann 2002), and reproduction (Karotam \& Oakeshott 1993) and resistance to pesticides ( $\mathrm{Li}$ et al. 2005). In insects, esterase genes have shown high rates of intraspecific and interspecific variation. Esterases are a very interesting group of enzymes because on one hand they are implicated in synaptogenesis, while on the other hand they are known to be involved in neuro-degeneration in adult tissue (Ahasan et al. 2009). Esterase variations have been reported in many animal and plant species, e.g. in the protozoan Tetrahymenal, maize (Schwartz 1960), swine (Augustinsson \& Olsson 1959), man (Harris et al. 1962) and insects. Numerous enzymatic activities also have been studied in Drosophila (Hubby 1963; Hubby \& Throckmorton 1965; Hubby \& Lewontin 1966; Lewontin \& Hubby 1966; Dickinson \& Sullivan 1975). All these studies have suggested that the esterase isozymes exhibit high level of polymorphism in Drosophila and other organisms, and this polymorphism offers adaptive flexibilities to these species.

Enzyme induction by environmental cues is potentially a key adaptive process. This environment dependent response may underlie phenomena such as physiological acclimation (Magnum \& Towle 1977) or morphological plasticity (Schlichting 1986). However, there are virtually no studies on enzymes in relation to the evolutionary significance of circadian rhythms. The above review of the literature has already showed that circadian rhythms have a physiological basis. Since esterases are a major group of enzymes which regulate many physiological activities, it is hypothesized that the circadian changes which occur due to different light regimes also influence the esterase pattern in regulating these rhythms. To verify this hypothesis the present work aims to analyse the effect of different light regimes in Drosophila. Variation in the alpha and beta esterase activities have been studied using polyacrylamide gel electrophoresis in light/dark (12L:12D), continuous light (LL) and continuous dark (DD) at 15 th and 30th generations. For this study, two species of the montium subgroup that are closely related, i.e. $D$. gangotrii and D. jambulina, and the most common species $D$. melanogaster, belonging to the melanogaster species group, were used. The members of montium are heterogeneous both morphologically (Lemeunier et al. 1986) and karyotypically (Bock \& Wheeler 1972; Baimai 1980; Shyamala \& Ranganath 1994; Suma \& Ranganath 1997; Shakunthala \& Ranganath 2007). Several reports in different disciplines of biology have revealed that species closely related, as Scouras (1995) feels 'system comprising species of close phylogenetic relationship as well as species rather distant phylogenetically from each other greatly facilitate biological analysis such system is offered by the montium a sub group of Drosophila'. The purpose of using these three species is to find out the differential or species-specific responses, if any, to different light regimes.

\section{Materials and methods}

Three species, D. melanogaster, D. gangotrii and D. jambulina were used for the present investigation. Isozyme profiles of both sexes were studied in three different light regimes - light/dark (12L:12D), continuous light (LL) and continuous dark (DD) - for 30 generations. The flies were maintained in an environmental chamber at constant temperature of $20 \pm 1{ }^{\circ} \mathrm{C}$ and $75 \%$ relative humidity with the above light regimes and one group was maintained in the normal light conditions in the laboratory (LP: lab populations) but at the same constant temperature of $20 \pm 1^{\circ} \mathrm{C}$. This group served as control. At the 15th and 30th generations five-day-old adult flies (five males and five females) were drawn from each of the above four groups and used for the enzyme assay. The enzyme profiles of alpha esterase $(\alpha$-est) and beta esterase ( $\beta$-est) were analysed.

\section{Polyacrylamide gel electrophoresis (PAGE-7.5\% native gel)}

The polyacrylamide gel electrophoretic technique described by Hegde (1979) was used with appropriate modifications for slab gel electrophoresis. The sample homogenate was prepared from five adult male and female flies separately with $40 \mu 1$ of $40 \%$ sucrose solution in an Eppendorf tube using a Knot's pestle. The samples were homogenized by keeping the set-up on ice and later centrifuged at $4000 \mathrm{rpm}$ for $5 \mathrm{~min}$ at $4{ }^{\circ} \mathrm{C} .7 .5 \%$ polyacrylamide gel was used for separation of enzyme fractions. An equal (15-20 $\mu 1)$ volume of supernatant was carefully loaded to each well. The first well was loaded with $1 \%$ bromophenol blue as a dye marker. After the sample application, electrophoresis was carried out at $4{ }^{\circ} \mathrm{C}$ with 50 volts for 1 hour and at 60 volts till the run was continued until the tracking dye migrated the entire length of the gel. Esterase isozymes were identified in the gels by following the procedure described by Hegde (1979) using $\alpha$ - or $\beta$-naphthyl acetate as substrates. After the appearance of bands, the gels were photographed. Different $\alpha$ - or $\beta$-esterase loci were identified following the procedure described by Hegde (1979). Accordingly three esterase loci, namely est-1, est-2 and est-3, were found in the present studies each with two alleles, one fast (F) and one slow (S).

\section{Results}

The results of the qualitative analysis of the alpha esterase $(\alpha$-est) and beta esterase ( $\beta$-est) enzymes using polyacrylamide gel electrophoresis are provided in Table 1 and Figures 1 and 2. The figures show the banding patterns of the three species under different light regimes and generations. It has been reported that there are three loci in Drosophila species, designated $\alpha$-est $1, \alpha$-est 2 and $\alpha$-est 3 (Naseerulla \& Hegde 1993; Barker et al. 1986; Sokal et al. 1987). Similarly, in the present research work Drosophila species showed three loci with slow moving and fast moving bands (alleles). 


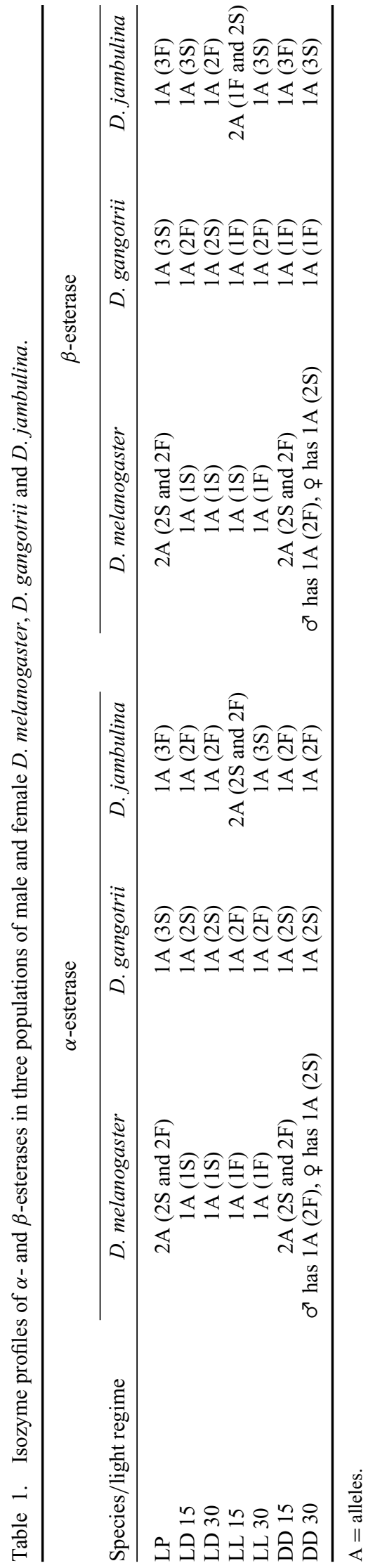

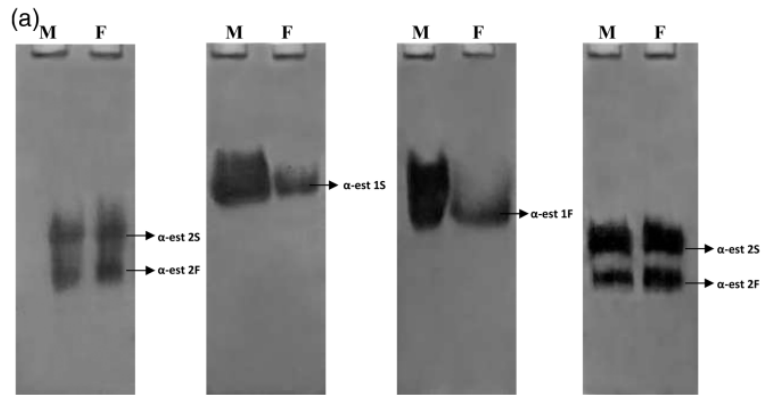
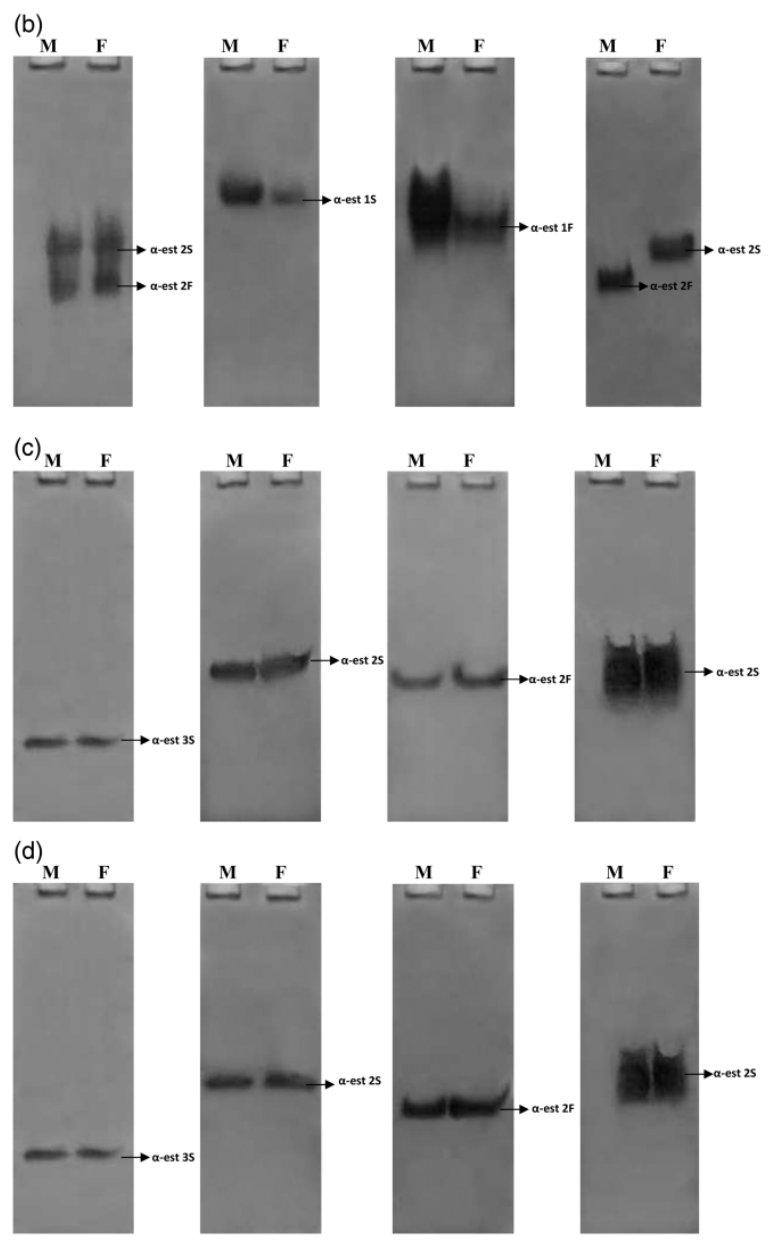

Figure 1. Alpha esterase profiles. Male and female $D$. melanogaster at (a) LP, LD 15, LL 15, DD 15; and (b) LP, LD 30, LL 30, DD 30; male and female D. gangotrii at (c) LP, LD 15, LL 15, DD 15; and (d) LP, LD 30, LL 30, DD 30; male and female D. jambulina at (e) LP, LD 15, LL 15, DD 15; and (f) LP, LD 30, LL 30, DD 30.

\section{Alpha esterase variation under different light regimes}

\section{D. melanogaster}

In LP, both the sexes of $D$. melanogaster showed an $\alpha$-est 2 slow moving band ( $\alpha$-est $2 S$ ) and an $\alpha$-est 2 fast moving band ( $\alpha$-est 2F). In LD 15 and LD 30 both male and female flies showed one allele each, i.e. $\alpha$-est $1 \mathrm{~S}$, whereas in LL 15 and LL 30 both male and female flies showed single band $(\alpha$-est $1 \mathrm{~F})$. However, in DD 15 as in LP, $\alpha$-est $2 \mathrm{~S}$ and 

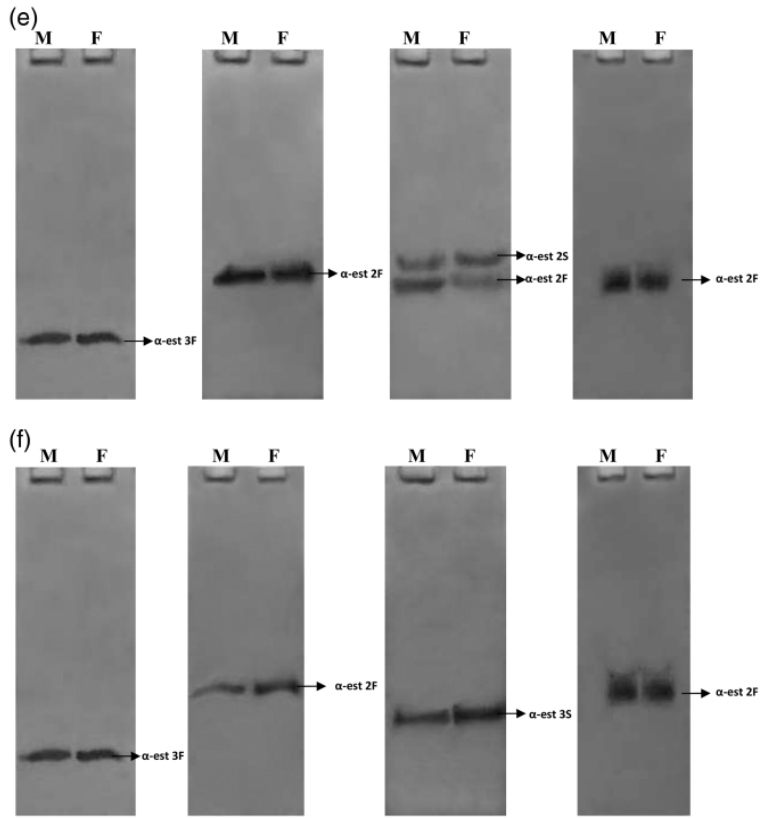

Figure 1. continued

$\alpha$-est $2 \mathrm{~F}$ were found both in males and females. At DD 30 male flies showed $\alpha$-est $2 \mathrm{~F}$ and females showed $\alpha$-est $2 \mathrm{~S}$ (Figure 1(a,b)).

\section{D. gangotrii}

$D$. gangotrii males and females showed $\alpha$-est $3 \mathrm{~S}$ in LP. In LD 15 and LD 30 both the sexes have $\alpha$-est 2S. In LL 15 and LL 30 male and female flies showed $\alpha$-est $2 \mathrm{~F}$, whereas DD 15 and DD 30 flies have $\alpha$-est $2 \mathrm{~S}$ (Figure 1(c, d)).

\section{D. jambulina}

In LP of this species, both the sexes showed $\alpha$-est 3F. At LD 15 and LD 30 male and female flies showed $\alpha$-est 2 F. At LL 15 two alleles were observed ( $\alpha$-est $2 \mathrm{~S}$ and $\alpha$-est $2 \mathrm{~F}$ ), whereas in LL 30, $\alpha$-est $3 \mathrm{~S}$ was observed. However, in DD 15 and DD 30 both the sexes have $\alpha$-est $2 \mathrm{~F}$ (Figure 1(e,f)).

\section{Beta esterase variation under different light regimes}

\section{D. melanogaster}

Both the sexes in laboratory populations showed two alleles ( $\beta$-est $2 \mathrm{~S}$ and $\beta$-est $2 \mathrm{~F}$ ). In LD 15 and LD 30 , male and female flies showed $\beta$-est $1 \mathrm{~S}$, whereas in LL $15, \beta$-est $1 \mathrm{~S}$ and in LL 30, $\beta$-est $1 \mathrm{~F}$ were observed. However, in DD 15, $\beta$-est $2 \mathrm{~S}$ and $\beta$-est $2 \mathrm{~F}$ were found both in males and females as in LP. At DD 30, males have $\beta$-est $2 \mathrm{~F}$ and females have $\beta$-est $2 \mathrm{~S}$ (Figure $2(\mathrm{a}, \mathrm{b})$ ).

\section{D. gangotrii}

$D$. gangotrii males and females showed $\alpha$-est $3 \mathrm{~S}$ in LP. Both the sexes have $\beta$-est $2 \mathrm{~F}$ and $\beta$-est $2 \mathrm{~S}$ respectively in LD 15 and LD 30. In LL 15 male and female flies showed $\beta$-est

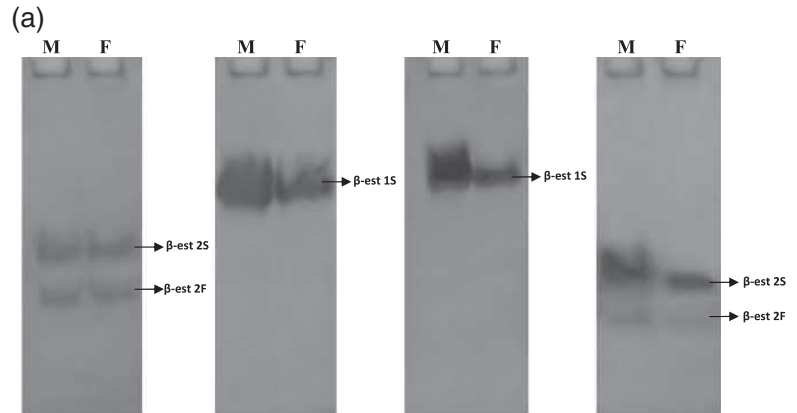

(b)

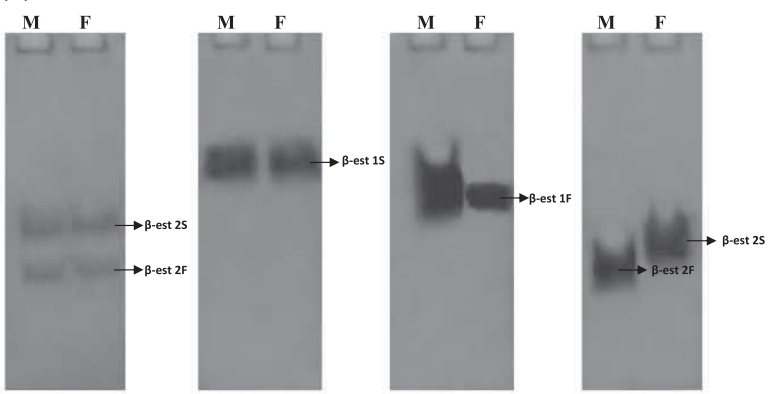

(c)

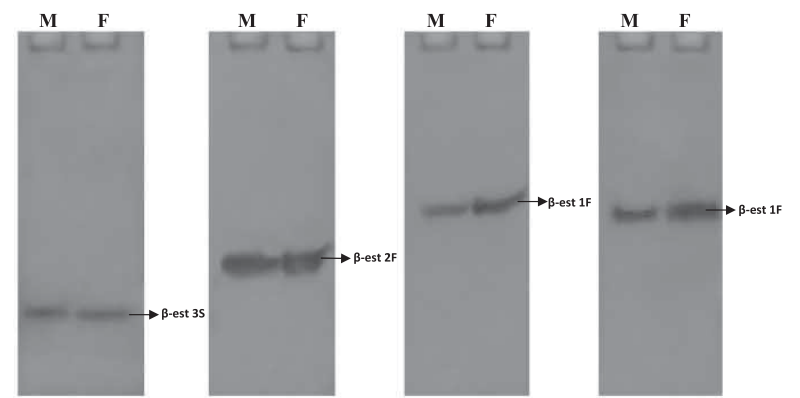

(d)

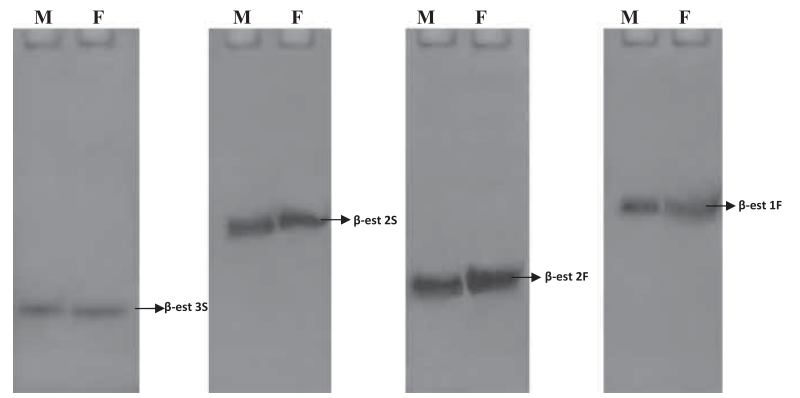

Figure 2. Beta esterase profiles. Male and female $D$. melanogaster at (a) LP, LD 15, LL 15, DD 15; and (b) LP, LD 30, LL 30 and DD 30; male and female D. gangotrii at (c) LP, LD 15, LL 15, DD 15; and (d) LP, LD 30, LL 30, DD 30; male and female D. jambulina at (e) LP, LD 15, LL 15, DD 15; and (f) LP, LD 30, LL 30, DD 30. 


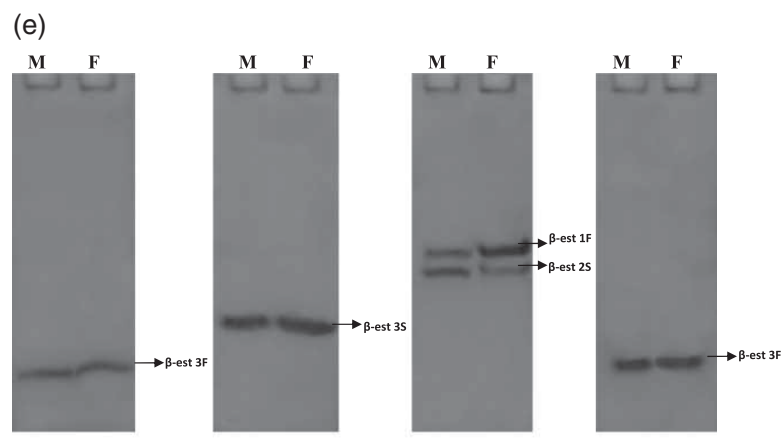

(f)

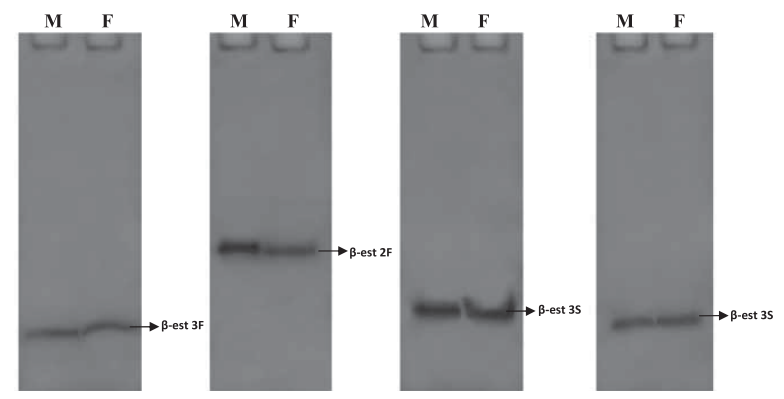

Figure 2. continued

$1 \mathrm{~F}$ and in LL 30 flies have $\beta$-est $2 \mathrm{~F}$, whereas in DD 15 and DD $30 \beta$-est $1 \mathrm{~F}$ was present (Figure 2(c, d)).

\section{D. jambulina}

In LP, both the sexes of $D$. jambulina showed $\beta$-est $3 \mathrm{~F}$. At LD 15 male and female flies showed $\beta$-est $3 \mathrm{~S}$ and in LD 30 both the sexes have $\beta$-est $2 \mathrm{~F}$. Two alleles were observed at LL 15 , those were $\beta$-est $1 \mathrm{~F}$ and $\beta$-est $2 \mathrm{~S}$, whereas in LL 30 only one allele was found, i.e. $\beta$-est $3 \mathrm{~S}$. However, in $\mathrm{DD} 15, \beta$-est $3 \mathrm{~F}$ and in DD $30, \beta$-est $3 \mathrm{~S}$ were present (Figure 2(e, f)).

\section{Discussion}

Esterases are a heterogeneous group of enzymes catalysing various esterification reactions. They are classified as nonglucose metabolizing enzymes (Gillespie \& Kojima 1968). Studies have demonstrated that the genetic loci encoding the synthesis of esterases have high variability (Naseerulla \& Hegde 1993). In the present investigation, there were variations in both alpha and beta esterase patterns in all three species employed, thus this study provides additional testimony for the esterase polymorphism that has been already reported. It has been noticed that there were three alpha esterase loci in Drosophila species which were designated as $\alpha$-est $1, \alpha$-est 2 and $\alpha$-est 3. Similarly there were three beta esterase loci which were designated as $\beta$-est $1, \beta$-est 2 and $\beta$-est 3. Each locus in turn had two alleles represented by a slow moving $(\mathrm{S})$ and fast moving alleles $(\mathrm{F})$. Investigations on the changes in enzyme activity in space and time among and within populations of Drosophila (Barker et al.
1986; Sokal et al. 1987) have been carried out. The overall number of esterase loci and alleles noticed in all the three species were also found in all these studies. However, the loci or the allele expressed at given light regime in these species exhibited variation.

The present study also shows that different light regimes change the pattern of expression of esterase alleles. For example, the pattern of expression of alpha and beta esterases in D. melanogaster, D. gangotrii and D. jambulina varied in different light regimes and at 15th and 30th generations. According to Sharma (2003) the changes in the light regime affect the physiology and also the rhythm of the fly. These physiological changes have reflected in terms of $\alpha$-esterase or $\beta$-esterase expression by switching over from one locus to another over generations. It was also observed that maintaining the flies in different light regimes can alter their allelic pattern. For example in D. jambulina, for $\alpha$-esterase, both at LL 15 and LL 30 different alleles were found when compared to other light regimes, however for $\beta$-esterase there were no similarities in allelic patterns in the light regimes. It has been shown that environmental changes can reduce the fitness of an organism (Hoffmann \& Parsons 1991). In the present situation subjecting the flies to different light regimes has altered the pattern of expression of alleles and hence their enzyme activity. There was no change in the expression of alleles from 15th to 30th generations in all light regimes except in LL, wherein there was change from 15 th to 30 th generation. In D. jambulina allelic variation was noticed in all light regimes when compared to LP. Flies maintained in different light regimes and generations showed variation in the expression of their alleles with respect to alpha esterase and beta esterase. But there were also certain similarities in alleles found at 15 th and 30th generations within the same species.

We observed variation in expression of alleles in the form of presence or absence of a given band in different light regimes. Some alleles were expressed in some light regimes but not in another regime. This shows that the different light regimes affect the expression of esterase alleles. As mentioned in the introduction, biological rhythms are endogenously generated self-sustaining mechanisms which are influenced by environmental stimuli such as light and feeding (Schibler 2005). The organism is able to quickly adjust or adapt to changing conditions by making appropriate physiological changes. The physiological change which is brought about by the change in the rhythm is evident in the present study because the esterase pattern was different in different light regimes. Thus this observation also confirms the hypothesis that circadian changes which occur due to different light regimes also influence the esterase pattern in regulating these rhythms.

The three species, D. melanogaster and the two species of the montium group showed differences in the expression of alleles at different light regimes. Although the montium subgroup is closely related to the melanogaster species group there was variation in terms of banding. This 
shows the differential response of different species to the light regimes. There was species specific difference which has some bearing on the general activity of these flies. $D$. melanogaster is a cosmopolitan domestic species and the flies are quite active with high fecundity and fitness in most environmental conditions (Bock \& Wheeler 1972). However, in the montium subgroup, both $D$. gangotrii and $D$. jambulina coexist in nature, and there were no similarities in their expression of alleles at different light regimes. However, these two species are more closely related to each other than to D. melanogaster. So the present study showed that the two montium species are closely related and are similar in their response to different light regimes.

\section{Acknowledgements}

We are grateful to the RFSMS Scheme, University Grant Commission, New Delhi and DOS in Zoology, University of Mysore for the facility.

\section{References}

Ahasan MM, Shahjahan RM, Begum RA, Sakina SZ, Jamal Uddin AFM. 2009. Esterase in different age group of farm chicken (Gallus gallus) with reference to the nervous tissue. Int J Sustain Agril Tech. 5:98-101.

Argentine JA, James AA. 1995. Characterization of a salivary gland-specific esterase in the vector mosquito Aedes aegypti. Ins Biochem Mol Biol. 25:621-630.

Augustinsson KB, Olsson B. 1959. Esterases in the milk and blood plasma of swine. II. Activities at different stages during the lactation and suckling periods, and plasma arylesterase as a gene-controlled enzyme. J Biochem. 71:484- 492.

Baimai V. 1980. Metaphase karyotypes of certain species of the Drosophila montium subgroup. Jpn J Genet. 55:165-175.

Barker JSF, East PD, Weir BS. 1986. Temporal and microgeographic variation in allozyme frequencies in a natural population of D. buzzatii. Genetics. 112:577-611.

Bitondi MMG, Mestriner MA. 1983. Esterase isozymes of Apis mellifera: Substrate and inhibition characteristics, developmental ontogeny, and electrophoretic variability. Biochem Genet. 21:985-1002.

Blau J, Young MW. 1999. Cycling vrille expression is required for a functional Drosophila clock. Cell. 99:661-671.

Bock IR, Wheeler MR. 1972. The Drosophila melanogaster species group. Genetics. 7:1-102.

Dickinson WJ, Sullivan DT. 1975. Gene-Enzyme Systems in Drosophila. New York: Berlin-Heidelberg-Springer.

Dvornyk V, Vinogradova O, Nevo E. 2003. Origin and evolution of circadian clock genes in prokaryotes. Proc Natl Acad Sci USA. 100:2495-2500.

Edery I, Zwiebel LJ, Dembinska ME, Rosbash M. 1994. Temporal phosphorylation of the Drosophila period protein. Proc Natl Acad Sci USA. 91:2260-2264.

Gillespie JH, Kojima K. 1968. The degree of the polymorphism in enzymes involved in energy production compared to that in nonspecific enzymes in two $D$. ananassae populations. Proc Natl Acad Sci USA. 61:582-585.

Harini BP. 2010. Correlation between mating propensity and productivity in few species of Drosophila exposed to light and dark cycle under laboratory environments. World J Zool. 5:306-313.
Harris H, Hopkinson DA, Robson EB. 1962. Two dimensional electrophoresis of pseudocholinestearse components in normal serum. Nature. 196:1296-1298.

Hegde SN. 1979. Studies on cyto-taxonomy and genetics of a few members of the melanogaster species group of Drosophila [Ph.D. thesis]. Mysore (India): University of Mysore.

Hendricks JC, Lu S, Kume K, Yin JCP, Yang Z, Sehgal A. 2003. Gender dimorphism in the role of cycle (BMAL1) in rest, rest regulation and longevity in Drosophila melanogaster. J Biol Rhythms. 18:12-25.

Hidayat P, Goodman WG. 1994. Juvenile hormone and hemolymph juvenile hormone binding protein titers and their interaction in the hemolymph of fourth stadium Manduca sexta. Ins Biochem Mol Biol. 24:709-715.

Hoffmann AA, Parsons PA. 1991. Evolutionary genetics and environmental stress. Oxford: Oxford University Press.

Hubby J. 1963. Protein differences in Drosophila. I. Drosophila melanogaster. Genetics. 48:871-879.

Hubby J, Lewontin RC. 1966. A molecular approach to the study of genic heterozygosity in natural populations. I. The number of alleles at different loci in Drosophila pseudoobscura. Genetics. 54:577-594.

Hubby J, Throckmorton LH. 1965. Protein differences in Drosophila. 11. Comparative species genetics and evolutionary problems. Genetics. 52:203-215.

Karotam J, Oakeshott JG. 1993. Regulatory aspects of esterase 6 activity variation in sibling Drosophila species. Heredity. 71:41-50.

Klarsfeld A, Rouyer F. 1998. Effects of circadian mutations and LD periodicity on the life span of Drosophila melanogaster. J Biol Rhythms. 13:471-478.

Kumar S, Mohan A, Sharma VK. 2005. Circadian dysfunction reduces lifespan in Drosophila melanogaster. Chronobiol Int. 22:641-653.

Lemeunier F, David JR, Tsacas L, Ashburner M. 1986. The melanogaster species group. In: Ashburner M, Carson HL, Thompson JN, editor. The genetics and biology of Drosophila. London: Academic Press; p. 147-256.

Lewontin RC, Hubby JL. 1966. A molecular approach to the study of the hetrozygosity in natural populations. II. Amount of variation and degree of heterozygosity in natural populations of Drosophila pseudoobscura. Genetics. 54:595-609.

Li Y, Pruett JH, Davey RB, George JE. 2005. Toxicological and biochemical characterization of coumaphos resistance in the San Roman strain of Boophilus microplus (Acari, Ixodidae). Pest Biochem Physiol. 81:145-153.

Magnum CP, Towle DW. 1977. Physiological adaptation to unstable environments. Science. 65:67-75.

Morton RA. 1993. Evolution of Drosophila insecticide resistance. Genome. 36:1-7.

Naseerulla MK, Hegde SN. 1993. Lack of correlation between mating activity and EST-1 polymorphism in three natural and laboratory populations of Drosophila bipectinata. DIS 71:257-259.

Pittendrigh CS, Minis DH. 1972. Circadian systems: Longevity as a function of circadian resonance in Drosophila melanogaster. Proc Natl Acad Sci USA. 69:15371539.

Schibler U. 2005. The daily rhythms of genes, cells and organs: Biological clocks and circadian timing in cells. EMBO Rep. 6:9-13.

Schlichting CD. 1986. The evolution of phenotypic plasticity in plants. Ann Rev Ecol Syst. 17:667-693.

Schwartz D. 1960. Genetic studies on mutant enzymes in maize: Synthesis of hybrid enzymes by heterozygotes. Proc Natl Acad Sci USA. 46:1210-1215. 
Scouras ZG. 1995. The Drosophila montium subgroup species: Recent cytogenetic molecular, development and evolutionary studies. Bios. 3:125-158.

Shakunthala V, Ranganath HA. 2007. Localization of heterochromatin of four species of the montium subgroup of Drosophila. Cytologia. 72:279-286.

Shanmugavelu M, Baytan AR, Chesnut JD, Bonning BC. 2000. A novel protein that binds juvenile hormone esterase in fat body tissue and pericardial cells of the tobacco hornworm Manduca sexta. J Biol Chem. 275:1802-1806.

Sharma VK. 2003. Adaptive significance of circadian clocks. Chronobiol Int. 20:901-919.

Sheeba V, Sharma VK, Chandrashekaran MK, Joshi A. 1999. Persistence of eclosion rhythm in populations of Drosophila melanogaster after 600 generations in an aperiodic environment. Naturwissenschaften. 86:448-449.

Sheeba V, Sharma VK, Shubha K, Chandrashekaran MK, Joshi A. 2000. The effect of different light regimes on adult life span in Drosophila melanogaster is partly mediated through reproductive output. J Biol Rhythms. 15: 380-392.
Shereen K, Shakunthala V. 2012. Eclosion behaviour of three species of Drosophila under different light regimes. Ind J Exp Biol. 50:660-664.

Shyamala BV, Ranganath HA. 1994. Karyotypic divergence due to heterochromatin among seven Indian species of the montium subgroup of Drosophila. The Nucleus. 37:46-52.

Sokal RR, Oden NL, Barker JSF. 1987. Spatial structure in Drosophila buzzatii populations: Simple and directional spatial autocorrelation. Am Nat. 129:122-42.

Suma OL, Ranganath HA. 1997. Characterization of heterochromatin in three species of the montium subgroup of Drosophila. Cytologia. 62:91-95.

Villatte F, Bachmann TT. 2002. How many genes encode cholinesterase in arthropods? Pest Biochem Physiol. 73:122129.

Xu K, Zheng X, Sehgal A. 2008. Regulation of feeding and metabolism by neuronal and peripheral clocks in Drosophila. Cell Metab. 8:289-300.

Zera AJ, Holtmeir CL. 1992. In vivo and in vitro degradation of juvenile hormone-III in presumptive long winged and short winged Gryllus rubens. J Ins Physiol. 38:61-74. 